\title{
Coffee polyphenols modulate whole-body substrate oxidation and suppress postprandial hyperglycaemia, hyperinsulinaemia and hyperlipidaemia
}

\author{
Takatoshi Murase*, Yuka Yokoi, Koichi Misawa, Hideo Ominami, Yasuto Suzuki, Yusuke Shibuya \\ and Tadashi Hase \\ Biological Science Laboratories, Kao Corporation, 2606 Akabane, Ichikai-machi, Haga-gun, Tochigi 321-3497, Japan
}

(Submitted 27 January 2011 - Final revision received 20 July 2011 - Accepted 11 August 2011 - First published online 29 September 2011)

\section{Abstract}

Postprandial energy metabolism, including postprandial hyperglycaemia, hyperinsulinaemia and hyperlipidaemia, is related to the risk for developing obesity and CVD. In the present study, we examined the effects of polyphenols purified from coffee (coffee polyphenols (CPP)) on postprandial carbohydrate and lipid metabolism, and whole-body substrate oxidation in $\mathrm{C} 57 \mathrm{BL} / 6 \mathrm{~J} \mathrm{mice}$. In mice that co-ingested CPP with a lipid-carbohydrate (sucrose or starch)-mixed emulsion, the respiratory quotient determined by indirect calorimetry was significantly lower than that in control mice, whereas there was no difference in $V_{\mathrm{O} 2}$ (energy expenditure), indicating that CPP modulates postprandial energy partitioning. CPP also suppressed postprandial increases in plasma glucose, insulin, glucose-dependent insulinotropic polypeptide and TAG levels. Inhibition experiments on digestive enzymes revealed that CPP inhibits maltase and sucrase, and, to a lesser extent, pancreatic lipase in a concentration-dependent manner. Among the nine kinds of polyphenols (caffeoyl quinic acids (CQA), diCQA, feruloyl quinic acids (FQA)) contained in CPP, di-CQA showed more potent inhibitory activity than CQA or FQA on these digestive enzymes, suggesting a predominant role of di-CQA in the regulation of postprandial energy metabolism. These results suggest that CPP modulates whole-body substrate oxidation by suppressing postprandial hyperglycaemia and hyperinsulinaemia, and these effects are mediated by inhibiting digestive enzymes.

Key words: Chlorogenic acid: Energy metabolism: Glucosidase: Hyperglycaemia: Polyphenols

The constellation of metabolic abnormalities, including visceral obesity, dyslipidaemia (high TAG and LDL-cholesterol or low HDL-cholesterol), hyperglycaemia and elevated blood pressure, is known as the metabolic syndrome, which increases the risk of developing $\mathrm{CVD}^{(1)}$. Postprandial hyperglycaemia and hyperlipidaemia induce endothelial dysfunction associated with increased oxidative stress and vascular inflammation, and are thus involved in the pathogenesis of atherosclerosis ${ }^{(2,3)}$. Therefore, pharmacological ${ }^{(4-6)}$ or non-pharmacological ${ }^{(3,7)}$ interventions have been carried out to reduce postprandial metabolic abnormalities and damage. The recent STOPNIDDM (Study to Prevent Non-Insulin-Dependent Diabetes Mellitus) trial demonstrated that daily intake of acarbose, which improves postprandial hyperglycaemia by inhibiting $\alpha$-glucosidase, reduces the risk of developing type 2 diabetes, hypertension and $\mathrm{CVD}^{(6,8)}$. Further, postprandial glucose and lipid concentrations influence postprandial hormone levels, such as insulin and glucose-dependent insulinotropic polypeptide $(\mathrm{GIP})^{(9,10)}$, which in turn may modify fuel utilisation and storage in various tissues ${ }^{(11)}$. On the basis of the hypothesis that a low-glycaemic meal, compared to a high-glycaemic meal, suppresses increases in postprandial serum glucose and insulin, thereby promoting fat oxidation and reducing fat storage, several studies have been conducted to compare the effects of different glycaemic meals. These studies, however, failed to demonstrate any clear effects on fuel partitioning, probably due to differences in serum insulin levels after meal loading that were insufficient to modify substrate oxidation ${ }^{(11)}$.

Several epidemiological studies ${ }^{(12,13)}$ suggest that coffee consumption helps prevent type 2 diabetes, liver diseases and Parkinson's disease. Many of the physiological effects of coffee can be attributed to caffeine, which is abundant in coffee ${ }^{(12)}$. On the other hand, coffee contains many phenolic compounds (coffee polyphenols (CPP)) such as caffeoyl quinic acids (CQA - 5-CQA is often called chlorogenic acid), di-CQA and feruloyl quinic acids (FQA). It is estimated that a 200-ml cup of roasted ground coffee contains 20-675 mg CPP, and the daily intake of CPP for a coffee drinker is as much as $1 \mathrm{~g}^{(14)}$. We recently reported that long-term intake of CPP attenuates diet-induced obesity by

Abbreviations: ACC, acetyl-COA carboxylase; CPP, coffee polyphenols; CQA, caffeoyl quinic acid; FQA, feruloyl quinic acid; GIP, glucose-dependent insulinotropic polypeptide; $\mathrm{IC}_{50}, 50 \%$ inhibited by $\mathrm{CPP}$; RQ, respiratory quotient; SREBP-1c, sterol regulatory element-binding protein 1c. 
increasing energy expenditure, possibly through the suppression of sterol regulatory element-binding protein 1c (SREBP1c) and the expression of related-molecules (acetyl-CoA carboxylase (ACC) 2, stearoyl-CoA desaturase-1, etc.) in the liver and adipose tissue ${ }^{(15)}$. In addition, 5-CQA is reported to suppress the postprandial increase in blood glucose levels after maltose or sucrose loading by inhibiting $\alpha$-glucosidases and glucose absorption in rats ${ }^{(16)}$. Moreover, Johnston et $a l .{ }^{(17)}$ reported that the consumption of coffee containing 5-CQA modifies gastrointestinal hormone secretion and glucose tolerance in human subjects, suggesting the regulatory effects of 5-CQA on postprandial serum energy substrate states. Little is known, however, about the effects of CPP on postprandial energy metabolism, and the relation of the effects with postprandial serum glucose and lipid dynamics.

In the present study, we examined the effect of CPP on serum components (i.e. glucose, insulin, GIP and TAG) after a single oral loading of lipid-carbohydrate-mixed emulsion and its relationship with fuel partitioning in mice. Further, we examined the inhibitory effects of nine kinds of polyphenols (i.e. CQA, di-CQA and FQA) purified from CPP on the activities of maltase, sucrase and lipase.

\section{Experimental methods}

\section{Preparation of coffee polyphenols}

A measure of $2 \mathrm{~kg}$ of ground roasted-coffee beans was extracted with hot water, and the extract was reduced to a powder using the spray-dry method. The extract $(270 \mathrm{~g})$ was then dissolved in 9 litres of water and applied to an aromatic-type adsorbent column (Sepabeads SP70; Mitsubishi Chemical, Tokyo, Japan). After washing the column with water, CPP were eluted with $0 \cdot 1 \% \mathrm{NaOH}$, followed by neutralisation with ion-exchange resin to weak acidic $\mathrm{pH}$.
The recovered CPP-containing solution was concentrated to an approximate solid content of $10 \%(\mathrm{w} / \mathrm{v})$, spray-dried and CPP was obtained ( $40 \mathrm{~g}$ ).

The composition of CPP was measured by HPLC. Total polyphenol content in the CPP was $77 \cdot 1 \%$ (the sum of each of the polyphenols described later). Caffeine was not detected. The composition of polyphenols was 3-CQA (5.9\%), 4-CQA (12.0\%), 5-CQA (24.6\%), 3-FQA (5.9\%), 4-FQA (4.3\%), 5-FQA (6.6\%), 3,4-di-CQA (6.9\%), 3,5-di-CQA (7.0\%) and 4,5 -di-CQA $(4 \cdot 1 \%)$. The structures of representative quinic acids are shown in Fig. 1.

\section{Purification and isolation of coffee polyphenols}

A total of nine kinds of quinic acid derivatives (i.e. CQA, diCQA and FQA) were isolated using a medium pressure chromatography system (Yamazen, Osaka, Japan) equipped with an Ultra Pack ODS-A-40D column, UV detector PREP-UV10V, fraction collector FR 50N, gradient mixer GR200, degasifier and pump PUMP-60A. CPP (2 g) dissolved in $20 \mathrm{ml}$ solution A (acetic acid-methanol-water, 1:20:80) was applied to the ODS column, and eluted with solution $\mathrm{A}$ and solution $\mathrm{B}$ (methanol) at a flow rate of $10 \mathrm{ml} / \mathrm{min}$ as follows: 0-100 min, solution A (100\%); 100-600 min, solution B $(0 \rightarrow 100 \%)$. The CPP 3-CQA, 3,4-di-CQA, 3,5-di-CQA and 4,5-di-CQA were isolated as single compounds in this process. Both the mixtures of 4-CQA, 5-CQA and 3-FQA, and 4-FQA and 5-FQA were further separated using a preparative HPLC system (LC-908; Japan Analytical Industry Company Limited, Tokyo, Japan) equipped with an Inertsil ODS-3 column (GL Science, Inc., Tokyo, Japan) using trifluoroacetic acid-methanol-water (1:300:700) as the eluent at a flow rate of $9 \mathrm{ml} / \mathrm{min}$. The elution pattern was monitored by measuring the absorbance at $325 \mathrm{~nm} .{ }^{1} \mathrm{H}-\mathrm{NMR}$ spectra were recorded in $\mathrm{CD}_{3} \mathrm{OD}$ on a JEOL $\alpha 500$ NMR spectrometer (JEOL, Tokyo, Japan).<smiles>O=C(O)/C=C/c1ccc(O)c(O)c1</smiles>

5-COA (5-caffeoyl quinic acid)<smiles>COc1cc(/C=C/C(=O)OC2CC(O)(C(=O)O)CC(O)C2O)ccc1O</smiles>

5-FQA

(5-feruloyl quinic acid)<smiles>O=C(C=Cc1ccc(O)c(O)c1)OC1CC(O)(C(=O)O)CC(OC(=O)C=Cc2ccc(O)c(O)c2)C1O</smiles>

3,5-diCQA

(3,5-dicaffeoyl quinic acid)

Fig. 1. Structures of representative quinic acid derivatives. 
The physical and spectroscopic data of the isolated compounds were in agreement with those reported in the literature $^{(18,19)}$.

\section{Animals and diets}

Male C57BL/6J mice obtained from Charles River (Kanagawa, Japan) at 7 weeks of age were maintained at $23 \pm 2^{\circ} \mathrm{C}$ under a $12 \mathrm{~h}$ light $-12 \mathrm{~h}$ dark cycle (lights on from $07.00-19.00$ hours) with commercial mouse food (CE-2; Clea Japan, Tokyo, Japan) and used for the oral lipid-carbohydrate load test at 8-9 weeks of age. The present study was approved by the Animal Care Committee of the Kao Corporation. All animal experiments followed the Guidelines for the Care and Use of Laboratory Animals of the committee.

\section{Preparation of the lipid-carbohydrate-mixed emulsion}

Lipid emulsion was prepared by sonicating $40 \%$ maize oil solution containing 1.6\% egg lecithin (Wako Pure Chemical Industries Limited, Osaka, Japan). Lipid-carbohydrate-mixed emulsion was prepared by combining $40 \%$ lipid emulsion and $40 \%$ sucrose or $40 \%$ autoclaved soluble starch (Wako) solution. Therefore, the final concentrations of lipid and carbohydrate in the emulsion were $20 \%$ each.

\section{Indirect calorimetry}

Energy metabolism studies were performed using a magnetictype mass spectrometric calorimeter Arco-2000 (Arco System, Inc., Chiba, Japan). Mice were randomly divided into four groups: lipid-starch emulsion control, lipid-starch emulsion + CPP, lipid-sucrose emulsion control and lipidsucrose emulsion + CPP groups ( $n 8$ per group). Following an $8 \mathrm{~h}$ fast, $200 \mathrm{mg} / \mathrm{kg}$ body weight of CPP solution or water was administered, immediately followed by either $4 \mathrm{~g} / \mathrm{kg}$ body weight of lipid-starch-mixed emulsion or lipidsucrose-mixed emulsion by gastric tube. $V_{\mathrm{O} 2}$ and $\mathrm{CO}_{2}$ production were then measured for $3 \mathrm{~h}$. During this time, data from each chamber were collected every $5 \mathrm{~min}$, with a settling time of $60 \mathrm{~s}$, a measurement time of $15 \mathrm{~s}$ and room air was used as the reference. The respiratory quotient (RQ) was calculated from the measured values of $V_{\mathrm{O} 2}$ and $\mathrm{CO}_{2}$ exhalation $\left(V_{\mathrm{CO} 2}\right)$. Data were normalised by body weight $(\mathrm{kg})$. Locomotor activity was measured using an automated motion analysis system (Actracer-2000; ARCO System, Inc.) that detects the amount of centroid fluctuation using a weighted transducer.

\section{Oral lipid-carbohydrate load test}

The mice were randomly divided into four groups: lipidstarch emulsion control, lipid-starch emulsion + CPP, lipidsucrose emulsion control and lipid-sucrose emulsion + CPP groups ( $n 10$ per group). Following an $8 \mathrm{~h}$ fast, $200 \mathrm{mg} / \mathrm{kg}$ body weight of CPP solution or water was administered, immediately followed by either $4 \mathrm{~g} / \mathrm{kg}$ body weight of a lipid-starch-mixed emulsion or a lipid-sucrose-mixed emulsion by gastric tube. Blood samples were obtained from the orbital sinus under anaesthesia with diethyl ether at 0, 10, 30, 60, 120 and $180 \mathrm{~min}$ after administration of the emulsion load, and the plasma was obtained.

\section{Blood analysis}

The blood glucose levels were measured by Accu-Check Aviva (Roche Diagnostics, Tokyo, Japan). The plasma TAG concentrations were determined using a Wako TG E-Test (Wako). The plasma insulin and GIP levels were measured using an insulin EIA kit (Morinaga, Yokohama, Japan) and rat/mouse GIP (total) ELISA kit (Linco Research, Inc., St Charles, MO, USA), respectively, according to the manufacturer's instructions.

\section{Enzyme assays}

Maltase and sucrase assays were conducted as described in a previous report, with minor modifications ${ }^{(20)}$. In brief, the inhibitory effect of CPP on maltase was assessed using an acetone extract from rat small intestine (Sigma-Aldrich, St Louis, MO, USA) and maltose as the substrate. The reaction was started by adding $50 \mu \mathrm{l}$ enzyme $(500 \mu \mathrm{g}$ protein in $50 \mathrm{~mm}$ phosphate buffer, $\mathrm{pH} 7 \cdot 0$ ) solution to a mixture of $1 \%$ maltose $(25 \mu \mathrm{l})$ and test compound $(25 \mu \mathrm{l})$. The reaction mixture was incubated for $15 \mathrm{~min}$ at $37^{\circ} \mathrm{C}$ and the reaction was stopped with $75 \mu \mathrm{l}$ of $0 \cdot 2-\mathrm{M}-\mathrm{NaOH}$ solution. The solution was neutralised with $75 \mu \mathrm{l}$ of $0 \cdot 2-\mathrm{M}$-acetic acid. In the control experiment, maltose was added after the neutralisation step instead of adding it when the reaction was started. A $2 \%$ sucrose solution was used to measure sucrase activity. The glucose concentration of the reaction mixture was determined using a Glucose C2 Test Wako (Wako).

Lipase activity was assessed using porcine pancreatic lipase (Sigma) and lipase kit S (DS Pharma Biomedical Company Limited, Osaka, Japan). The enzyme was dissolved in $125 \mathrm{~mm}$-Tris- $\mathrm{HCl}$ buffer ( $\mathrm{pH} 7.5)$ at a concentration of $50 \mu \mathrm{g} / \mathrm{ml}$. After incubating the reaction mixture, which comprised enzyme solution $(8 \mu \mathrm{l})$, test compound $(30 \mu \mathrm{l})$, esterase inhibitor $(2 \mu \mathrm{l})$ and 5,5-dithiobis (2-nitrobenzoic acid; colour coupler; $100 \mu \mathrm{l}$ ), for $5 \mathrm{~min}$ at $30^{\circ} \mathrm{C}$, substrate was added and the mixture was incubated for an additional $30 \mathrm{~min}$ at $30^{\circ} \mathrm{C}$. The reaction was terminated by adding stop solution $(200 \mu \mathrm{l})$, and the absorbance was measured at $412 \mathrm{~nm}$ to quantify the reactive products.

\section{Statistical analysis}

All values are presented as mean values with their standard errors. Expired gas data (i.e. $V_{\mathrm{O} 2}$ and RQ) and plasma biochemical data (i.e. glucose, insulin, GIP and TAG) in the oral lipid-carbohydrate load test were analysed by repeated-measures ANOVA with factors of group and time, and the interaction of the factors. The differences between groups in plasma biochemical data were calculated as area under the curve and were assessed with the Student's $t$ test. A $P$ value of less than 0.05 was considered significant. Statistical analyses were conducted using the SAS software, version 9.2 (SAS Institute, Inc., Cary, NC, USA). 


\section{Results}

\section{Effect of coffee polyphenols on energy metabolism}

We extracted the data from 10 to $120 \mathrm{~min}$ of $V_{\mathrm{O} 2}$ and $\mathrm{RQ}$, as the data from 0 to $10 \mathrm{~min}$ were unstable due to experimental manipulations. Differences in $V_{\mathrm{O} 2}$ were not significantly different between the control and CPP groups after administering the lipid and sucrose-mixed emulsion (Fig. 2(a)). In contrast, CO-administration of CPP significantly reduced the emulsioninduced increase in the RQ value (Fig. 2(b); $P<0.05$, group factor in repeated-measures ANOVA). When the lipid and starch-mixed emulsion was administered, CPP similarly reduced the increase in RQ (Fig. 2(d); $P<0.05$, group factor). Locomotor activity did not significantly differ between the control and CPP groups (data not shown).

\section{Effect of coffee polyphenols on postprandial increase in glucose, insulin, glucose-dependent insulinotropic polypeptide and TAC}

Co-administration of CPP with a lipid and sucrose-mixed emulsion significantly reduced the increase in plasma glucose level (Fig. 3(a); $P<0 \cdot 05$, group $\times$ time interaction in repeated-measures ANOVA). CPP administration significantly suppressed the peak glucose level at $10 \mathrm{~min}$, although there was no difference in the total amount of glucose absorption calculated as area under the curve (Fig. 5(a)). The increase in insulin levels after emulsion administration was also suppressed by CPP (Fig. 3(b); $P<0 \cdot 05$, group $\times$ time interaction), concomitant with suppression of the increase in GIP (Fig. 3(c); $P<0.05$, group $\times$ time interaction) that is released from intestinal endocrine cells in response to luminal glucose or fat, and stimulates insulin secretion from pancreatic $\beta$-cells. Total GIP secretion, calculated as the area under the curve, was significantly smaller in the CPP group than in the control group (Fig. 5(c)). Plasma TAG levels peaked at $60 \mathrm{~min}$ after emulsion administration, and the increase was significantly suppressed when CPP was coadministered with the emulsion (Fig. 3(d); $P<0.05$, group $\times$ time interaction).

Plasma glucose exhibited a biphasic response to the lipid and starch-mixed emulsion administration, with a peak at 10 and $60 \mathrm{~min}$ (Fig. 4(a)). The peak value of glucose at $10 \mathrm{~min}$ was significantly lower in the CPP-administered group. CPP administration resulted in rightward shift of the peak insulin level, and the insulin level at $10 \mathrm{~min}$ was also significantly reduced (Fig. 4(b); $P<0 \cdot 05$, group $\times$ time interaction). CPP administration also significantly suppressed the increase in GIP levels (Fig. 4(c); $P<0 \cdot 05$, group $\times$ time interaction), with a significant reduction of total GIP secretion (Fig. 5(g)). Plasma TAG levels were also lower in the CPP group than in the control group at 10 and $30 \mathrm{~min}$ (Fig. 4(d)).

\section{Effect of coffee polyphenols on maltase, sucrase and lipase activities}

To clarify the anti-hyperglycaemic and anti-hyperinsulinaemic effects of CPP, inhibitory effects of various concentrations of
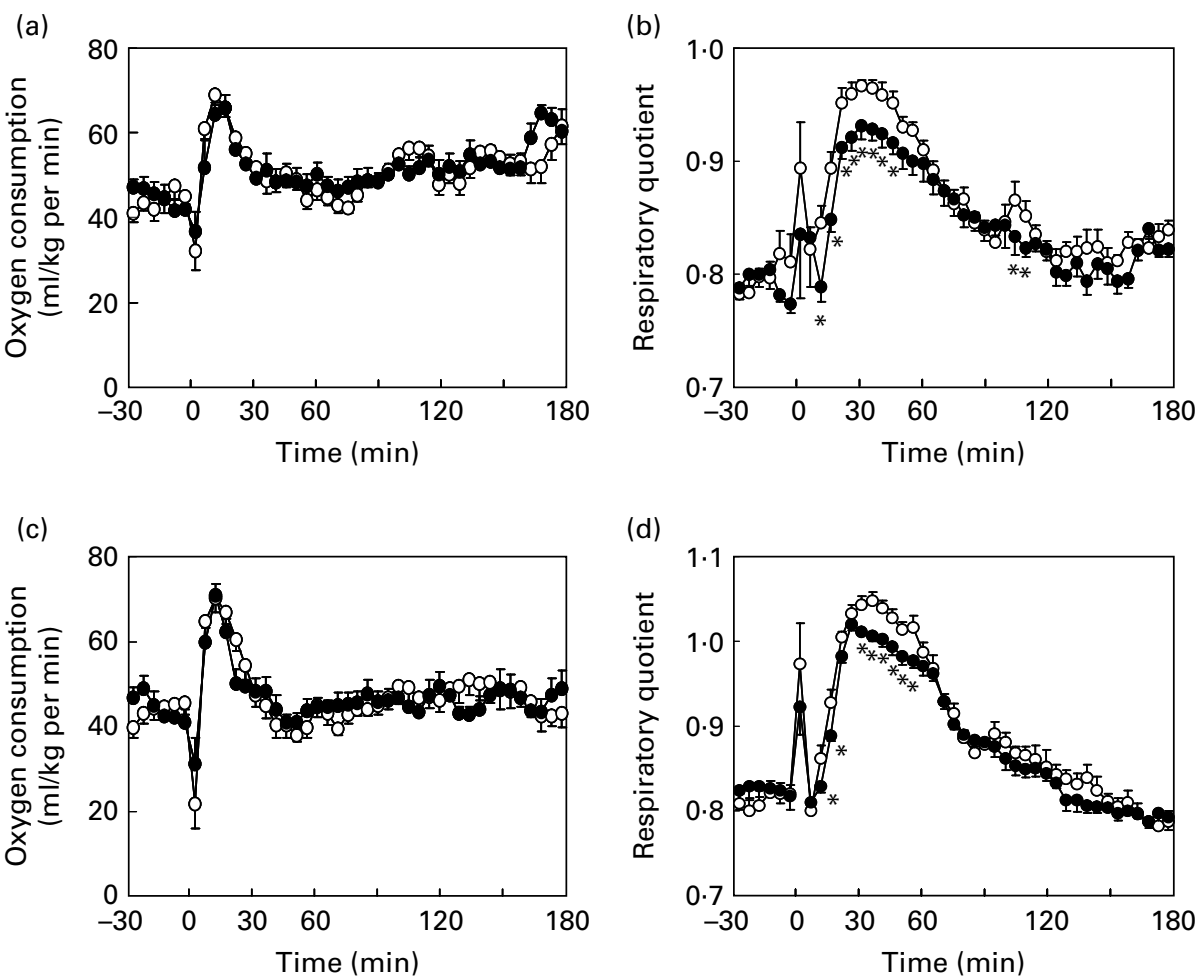

Fig. 2. Effect of coffee polyphenols (CPP) on whole-body energy metabolism. $V_{\mathrm{O} 2}$ and carbon dioxide exhalation were monitored for $3 \mathrm{~h}$ after administering $(a$ and $b$ ) lipid and sucrose-mixed emulsion or ( $c$ and d) lipid and starch-mixed emulsion, and the respiratory quotient was calculated. Values are means with their standard errors of eight mice. * Mean values were significantly different control group at each time point $(P<0.05)$. $-\bigcirc-$, Control; $-\bullet-$, CPP. 
(a)

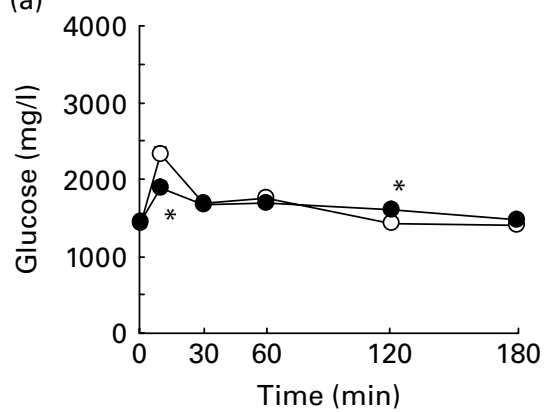

(c)

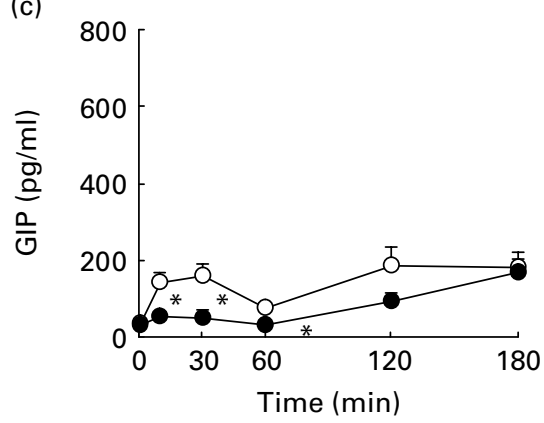

(b)

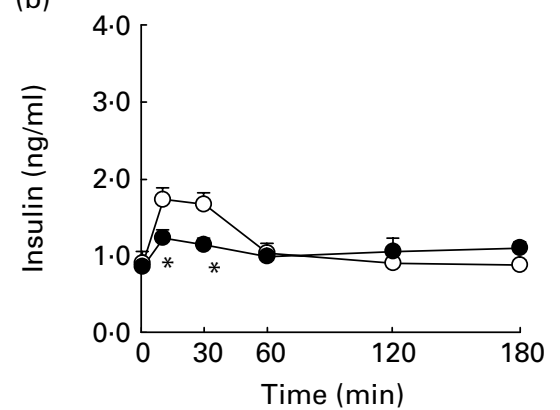

(d)

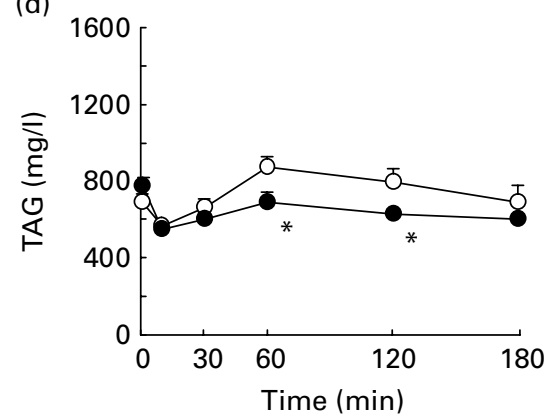

Fig. 3. Effects of coffee polyphenols (CPP) on plasma glucose, insulin, glucose-dependent insulinotropic polypeptide (GIP) and TAG concentrations after administration of a lipid and sucrose-mixed emulsion. Following an $8 \mathrm{~h}$ fast, $200 \mathrm{mg} / \mathrm{kg}$ body weight of CPP solution was administered, followed by lipid-sucrose-mixed emulsion by a gastric tube. At the indicated time, blood samples were obtained from the orbital sinus and (a) plasma glucose, (b) insulin, (c) GIP and (d) TAG levels were determined. * Mean values were significantly different control group at each time point $(P<0 \cdot 05)$. $-O-$, Control; $-\bullet-$, CPP.

CPP on maltase, sucrase and lipase activities were examined in vitro. CPP inhibited maltase activity in a concentrationdependent manner and approximately $0.07 \mathrm{mg} / \mathrm{ml} \mathrm{CPP}$ inhibited $50 \%\left(\mathrm{IC}_{50}\right)$ of the maltase activity (Fig. 6(a)). CPP also
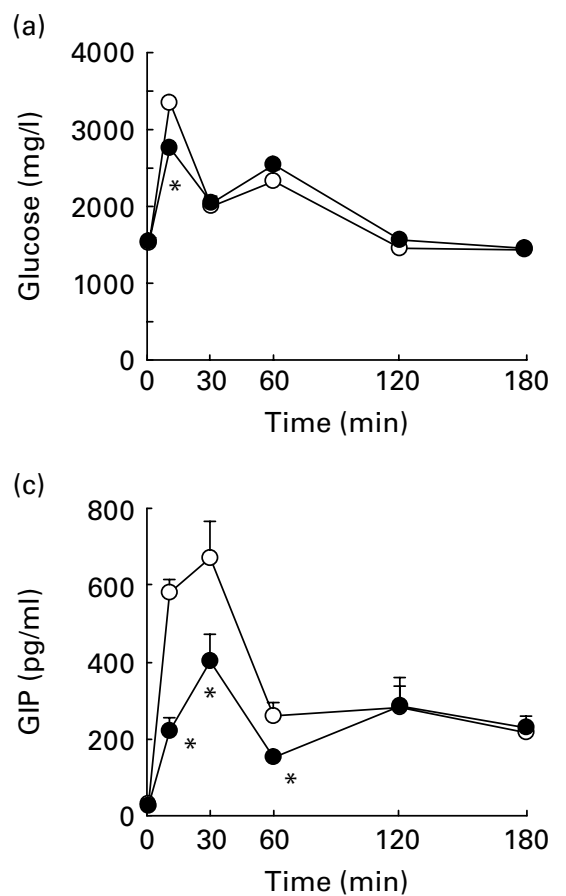

showed a concentration-dependent inhibition of sucrase activity, with an approximate $\mathrm{IC}_{50}$ of $0.35 \mathrm{mg} / \mathrm{ml}$ (Fig. 6(b)) Pancreatic lipase activity was inhibited by CPP with an approximate $\mathrm{IC}_{50}$ of $2.49 \mathrm{mg} / \mathrm{ml}$ (Fig. 6(c)).

(b)

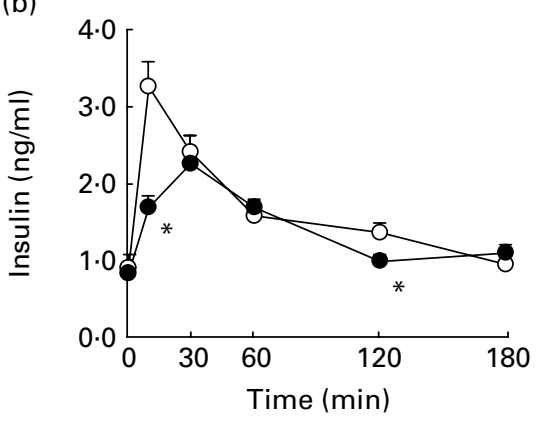

(d)

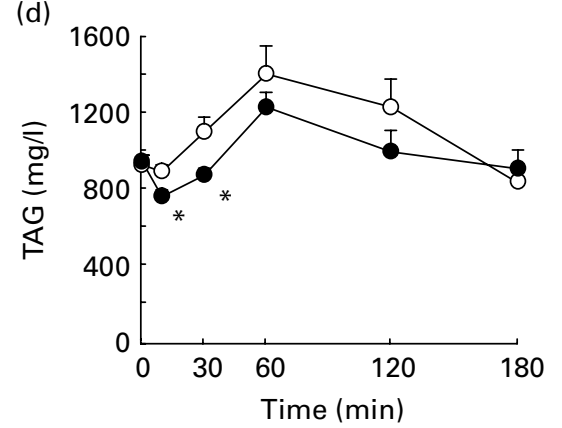

Fig. 4. Effects of coffee polyphenols (CPP) on plasma glucose, insulin, glucose-dependent insulinotropic polypeptide (GIP) and TAG concentrations after administration of a lipid and starch-mixed emulsion. Following an $8 \mathrm{~h}$ fast, $200 \mathrm{mg} / \mathrm{kg}$ body weight of CPP solution was administered, followed by lipid-starch-mixed emulsion by a gastric tube. At the indicated time, blood samples were obtained from the orbital sinus and (a) plasma glucose, (b) insulin, (c) GIP and (d) TAG levels were determined. ${ }^{*}$ Mean values were significantly different control group at each time point $(P<0 \cdot 05)$. $-O-$, Control; $-\bullet-$, CPP. 

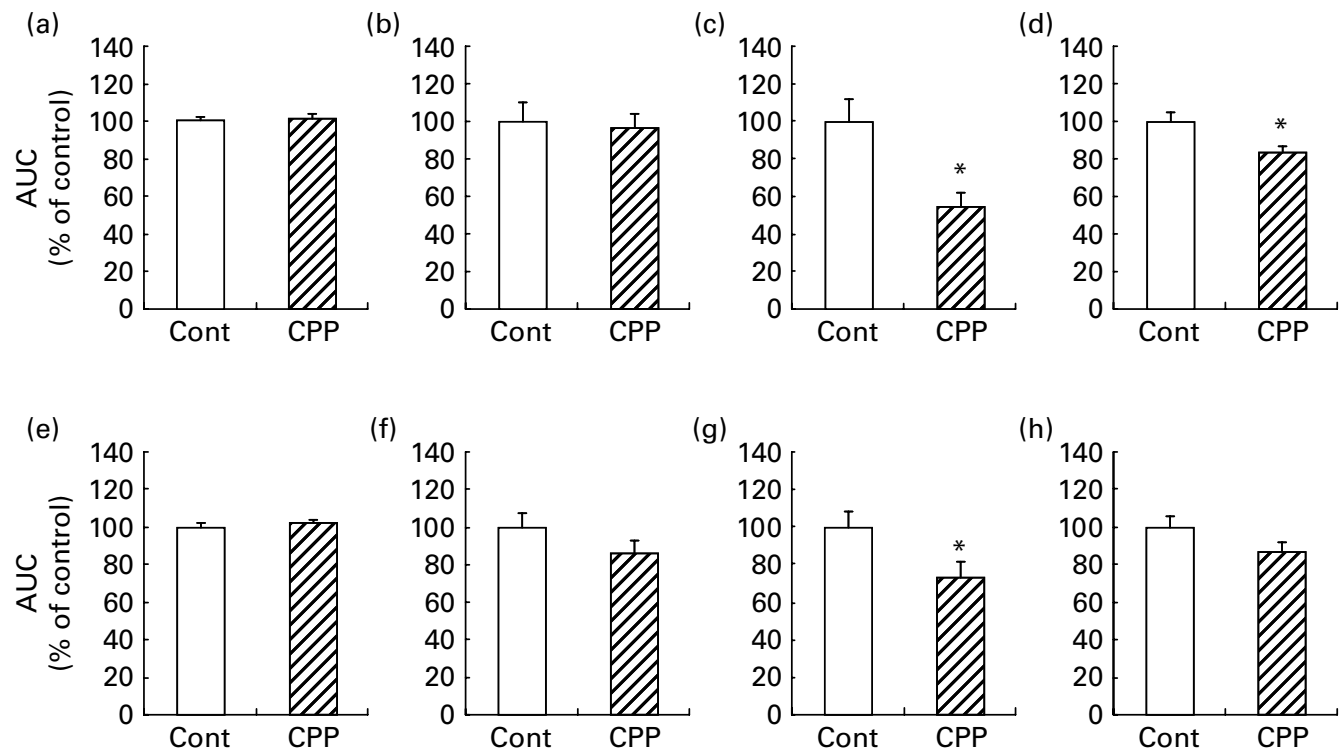

Fig. 5. Area under the curve (AUC) of plasma glucose, insulin, glucose-dependent insulinotropic polypeptide and TAG concentrations. (a-d) AUC of mice administered a lipid-sucrose-mixed emulsion. (e-h) AUC of mice administered a lipid-starch-mixed emulsion. Values are means with their standard errors of eight mice. * Mean value was significantly different from that of the control (Cont) group $(P<0.05$ ( $t$ test)). CPP, coffee polyphenols.

\section{Structure-activity relationship of coffee polyphenols on enzyme inhibitory action}

CPP comprises mainly nine types of caffeic acid derivatives (three CQA, three di-CQA and three FQA). In addition, approximately two-thirds of ingested chlorogenic acid reaches the colon, where it is likely to be hydrolysed to caffeic acid and quinic acid by the colonic microflora ${ }^{(21,22)}$. Therefore, to further clarify the molecular specificity of the polyphenols in $\mathrm{CPP}$ and the active compounds, we compared the effects of the nine molecules contained in CPP and three possible metabolites (i.e. caffeic acid, quinic acid and ferulic acid) on enzyme activities.

On the basis of the concentration dependency of CPP on enzyme inhibition (Fig. 6), the maltase- and sucrase-inhibitory activity of each sample was assessed at 0.05 and $0.30 \mathrm{mg} / \mathrm{ml}$, respectively. The three di-CQA (3,4-, 3,5- and 4,5-di-CQA) showed $35-83 \%$ inhibitory activity on maltase, whereas CQA and feruloyl quinic acids showed no marked activity at this concentration (Fig. 7(a)). The three CQA (3-, 4- and 5-CQA) and di-CQA (3,4-, 3,5- and 4,5-di-CQA), all of which have one or two caffeic acid moieties, inhibited sucrase activity by $32-46$ and $68-76 \%$, respectively (Fig. 7(b)). The FQA had a smaller inhibitory effect on sucrase activity. Among the building units of CQA and FQA, caffeic acid and ferulic acid inhibited sucrase activity, whereas quinic acid had no effect at the concentrations examined. Similarly, lipase activities were inhibited by $39-66 \%$ by di-CQA; however, CQA and FQA had no marked inhibitory activity at the concentrations (Fig. 7(c))

\section{Discussion}

In the present study, we examined the effects of CPP on serum components after a single oral loading of lipid-carbohydrate-mixed emulsion and its relationship with whole-body energy metabolism in mice. The present results suggest that CPP modulates whole-body substrate oxidation by suppressing postprandial hyperglycaemia and hyperinsulinaemia, and these effects are mediated by the inhibition of digestive enzymes.

The health benefits of phenolic compounds in plants have received increasing attention ${ }^{(23,24)}$, and the beneficial effects of coffee and its constituents have also been examined ${ }^{(12,13)}$. Coffee contains a large amount of caffeine in addition to phenolic compounds, including 5-CQA, and many of its physiological actions are assumed to be due to or influenced by caffeine ${ }^{(12,25)}$. In the present study, to clarify the biological function of polyphenols in coffee, we purified and examined the effects of CPP. Postprandial glycaemic responses are greatly modified by the co-ingestion of lipids ${ }^{(\mathcal{O}, 10)}$. In addition, carbohydrate is usually ingested together with lipid in daily life; therefore, we analysed the influence of CPP intake on postprandial responses after ingestion of lipid and carbohydrate.

Although there was no difference in energy expenditure, the increase in the RQ value after administering a lipid and carbohydrate-mixed emulsion was significantly reduced by CPP CO-administration, indicating that postprandial lipid utilisation is greater in mice administered CPP. Acute postprandial energy utilisation is greatly influenced by glucose and glucoseinduced hormone levels. Carbohydrates such as starch and sucrose are hydrolysed by digestive enzymes to form glucose, which induces the secretion of insulin from pancreatic $\beta$-cells, concomitant with the induction of incretin secretion, including GIP and glucagon-like peptide, from the intestinal tract. Insulin enhances lipogenesis by activating ACC-1, together with the suppression of lipolysis in adipose tissue through phosphorylation and subsequent inhibition of hormone-sensitive lipase $^{(26,27)}$. Further, insulin increases cellular malonyl-CoA, 

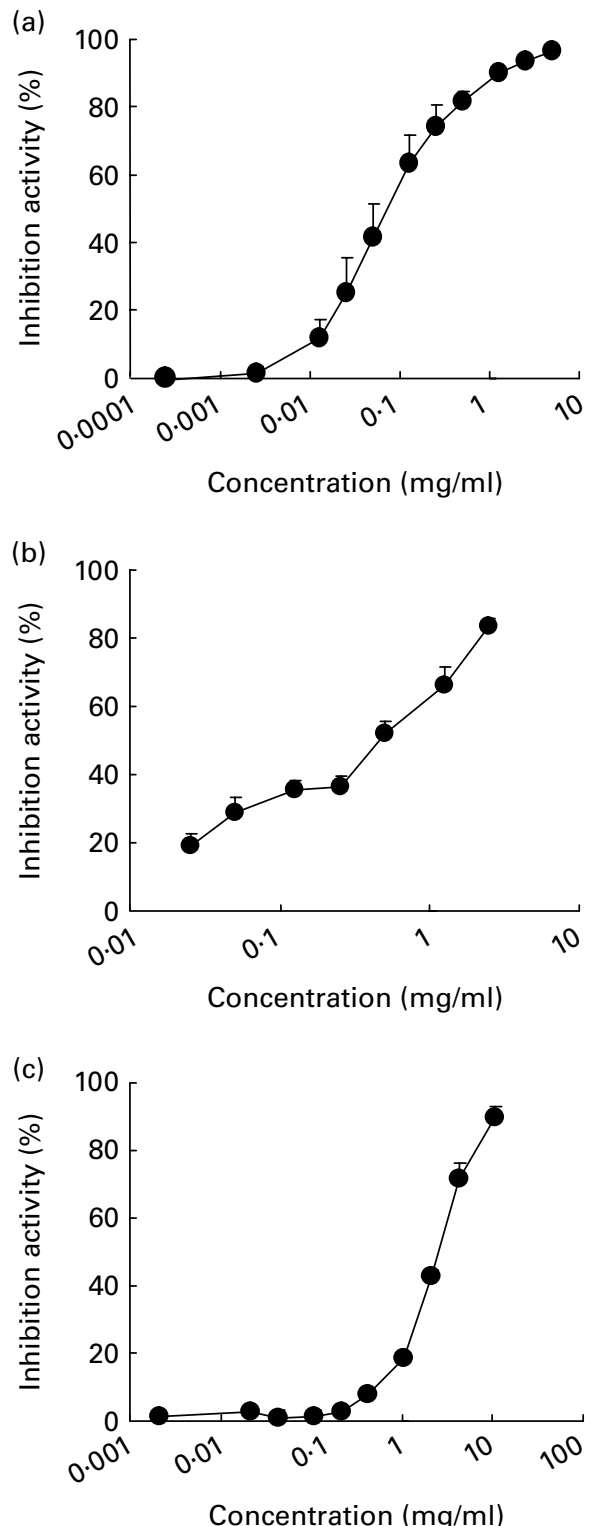

Fig. 6. Effects of coffee polyphenols (CPP) on maltase, sucrase and lipase activities. The inhibitory effect of CPP on (a) maltase was assessed using an acetone extract from rat small intestine and maltose as a substrate. (b) Sucrose solution was used for the measurement of sucrase activity. The glucose concentration of the reaction mixture was determined using a glucose assay kit and inhibition activity was calculated. (c) Lipase activity was assessed using porcine pancreatic lipase and a commercial kit. Values are means with their standard errors $(n 3)$.

which inhibits carnitine palmitoyl transferase- 1 by activating ACC- $2^{(28,29)}$, leading to a decrease in fatty acid oxidation. Thus, the increase in the serum insulin levels after carbohydrate ingestion influences energy partitioning by both increasing glucose utilisation through the stimulation of glucose incorporation to peripheral tissues and by suppressing fatty acid oxidation. Moreover, glucose-induced insulin secretion is enhanced by lipid, which is partly mediated by increased GIP secretion $^{(9,10)}$. Co-ingestion of CPP with lipid and carbohydrate-mixed emulsion suppressed the increase in serum GIP, which was consistent with the serum glucose and insulin levels, suggesting that $\mathrm{CPP}$ decreases the rate of intestinal absorption of glucose and subsequent serum hormone levels, thereby decreasing glucose utilisation and increasing lipid utilisation. Because suppression of the postprandial increase in serum glucose and the resulting changes in energy partitioning were observed both after starch and sucrose intake, these changes were at least partly mediated by the inhibitory action of CPP on glucosidases. In addition to 5-CQA, which has an inhibitory action on $\alpha$-glucosidase ${ }^{(17)}$, CPP contains a variety of polyphenolic compounds such as CQA isomers, di-CQA and FQA; therefore, we purified and examined the specificity of each compound on digestive
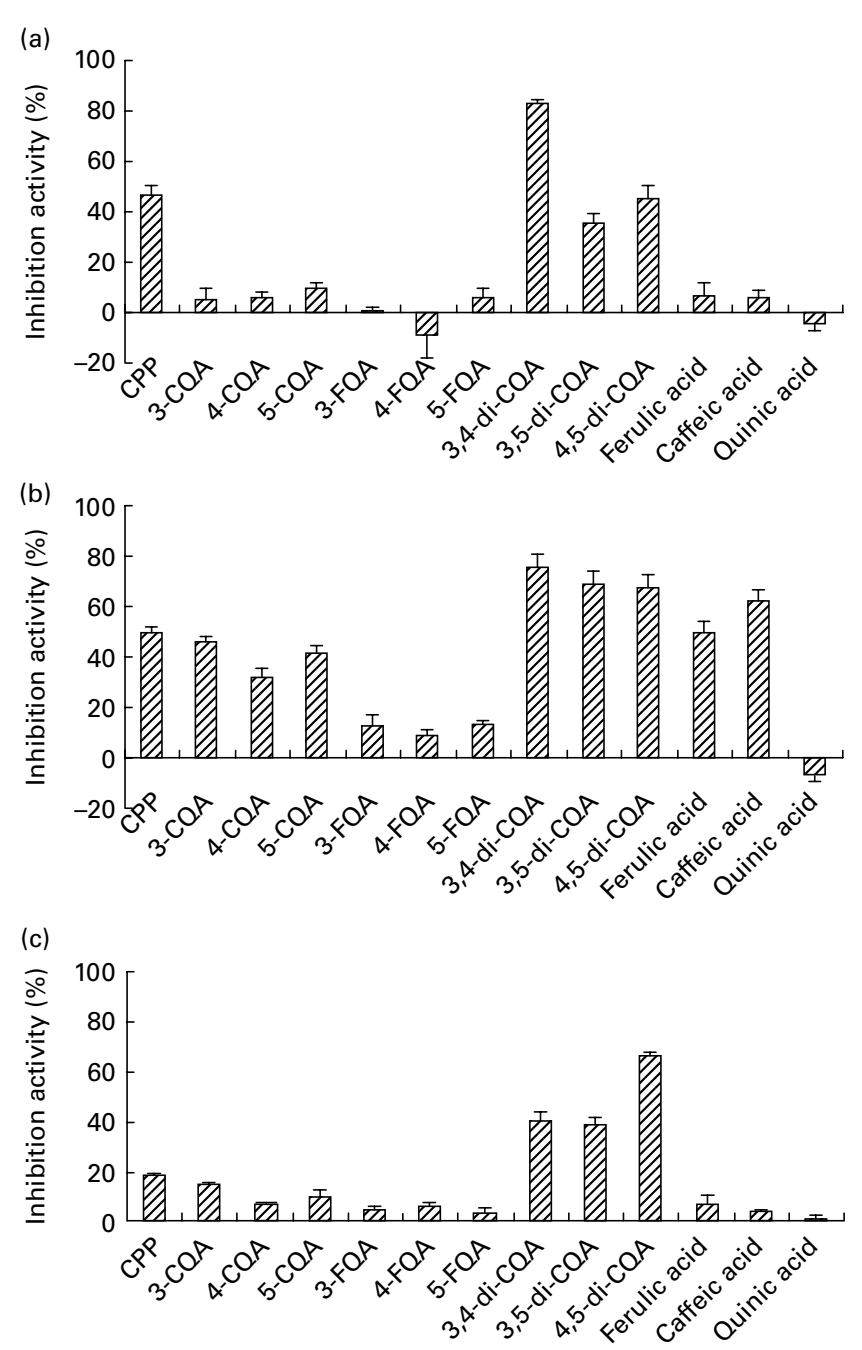

Fig. 7. Structure-activity relationship of coffee polyphenols (CPP) and their constituents on enzyme inhibitory action. The inhibitory effect of CPP and their constituents on (a) maltase was assessed using an acetone extract from rat small intestine and maltose as a substrate. (b) Sucrose solution was used for the measurement of sucrase activity. The glucose concentration of the reaction mixture was determined by glucose assay kit and inhibition activity was calculated. (c) Lipase activity was assessed using porcine pancreatic lipase and a commercial kit. Maltase, sucrase and lipase inhibitory activity of each sample was assessed at $0.125,0.75$ and $5.0 \mathrm{~mm}$, respectively. Maltase, sucrase and lipase activities of CPP were assessed at 0.05, 0.30 and $2.00 \mathrm{mg} / \mathrm{ml}$, respectively. Values are means with their standard errors $(n 3)$. CQA, caffeoyl quinic acids; FQA, feruloyl quinic acids. 
enzyme inhibition. CPP exhibited more potent inhibitory action on maltase than sucrase, and, among the quinic acid derivatives, di-CQA most strongly inhibited maltase. CQA and di-CQA also inhibited sucrase activity, and FQA did not show an inhibitory effect at the concentrations examined. These results suggest that CQA and di-CQA largely contribute to the inhibition of glucosidases, and the subsequent suppression of postprandial serum glucose and the insulin increase, which resulted in the changes in fuel partitioning. The present results indicate that caffeoyl group(s) bound to a quinic acid molecule has an important role in the enzyme inhibitory effects, and the inhibitory activity increases with an increasing number of caffeoyl groups. Differences in molecular size, conformation or physical properties, including lipophilicity/ hydrophilicity $^{(30)}$, of CQA might affect the affinity of CQA for enzymes and subsequent inhibitory actions. The precise mechanisms by which di-CQA exert more potent inhibitory effects require further investigation.

In addition to glucosidase inhibition, CPP also inhibited pancreatic lipase and decreased the increase in serum TAG after loading of a lipid and carbohydrate-mixed emulsion. Lipase activity was preferably inhibited by di-CQA, suggesting the contribution of this molecular species to the suppression of the postprandial TAG increase. Postprandial lipid oxidation is primarily determined by carbohydrates, rather than by the amount of fat consumed, when fat is consumed together with carbohydrate ${ }^{(31)}$; therefore, it is conceivable that the CPP-induced decrease in the serum glucose and insulin levels, rather than the decrease in the TAG levels, were more clearly reflected by the RQ.

Recently, we reported that CPP alleviates diet-induced obesity ${ }^{(15)}$. Habitual intake of CPP suppresses mRNA expression of lipogenic enzymes including fatty acid synthase, stearoyl-CoA desaturase-1 and ACC-1 by downregulating SREBP-1c. In addition, CPP increases fatty acid oxidation, concomitant with a reduction of ACC-2 mRNA and ACC activity, and decreases cellular malonyl-CoA levels. Because the expression of SREBP-1c is stimulated by glucose or insulin ${ }^{(32,33)}$, suppression of SREBP-1c expression and subsequent reduction of body fat accumulation might be partly mediated by the CPP-induced lowering effect of postprandial insulin.

GIP exerts various effects on adipocytes, including stimulating lipoprotein lipase activity ${ }^{(34)}$, fatty acid uptake ${ }^{(35)}$ and lipogenesis $^{(36)}$. These studies suggest an important role of GIP in the regulation of fatty acid metabolism and fat deposition, and its physiological significance is emphasised by the fact that GIP receptor knockout mice fed a high-fat diet are obesity resistant ${ }^{(37)}$. Given the effects of GIP on adipocytes, suppression of the postprandial GIP increase by CPP might contribute not only to altered energy partitioning but also to reduced body fat accumulation through attenuation of the action of GIP against adipose tissue.

In summary, CPP modulates whole-body lipid and carbohydrate oxidation by suppressing postprandial glucose and insulin increases through inhibiting digestive enzymes including maltase and sucrase. Structure-activity relationship studies revealed that di-CQA have more potent inhibitory activities on digestive enzymes, suggesting the contribution of the species to the effects of CPP.

\section{Acknowledgements}

The authors thank Tohru Yamaguchi for help in the statistical analysis. T. M. designed the study and wrote the manuscript. Y. Y. and K. M. conducted the animal or enzymatic experiments and analysed the data. H. O., Y. Su. and Y. Sh. performed the purification of the quinic acid derivatives. T. H. helped to design the study. The authors declare no conflict of interest. No external funding was received for the present study.

\section{References}

1. Després JP, Lemieux I, Bergeron J, et al. (2008) Abdominal obesity and the metabolic syndrome: contribution to global cardiometabolic risk. Arterioscler Thromb Vasc Biol 28, 1039-1049.

2. Kanter JE, Johansson F, LeBoeuf RC, et al. (2007) Do glucose and lipids exert independent effects on atherosclerotic lesion initiation or progression to advanced plaques? Circ Res $\mathbf{1 0 0}$, 769-781.

3. O'Keefe JH \& Bell DS (2007) Postprandial hyperglycemia/ hyperlipidemia (postprandial dysmetabolism) is a cardiovascular risk factor. Am J Cardiol 100, 899-904.

4. Guerciolini R (1997) Mode of action of orlistat. Int J Obes Relat Metab Disord 21, Suppl. 3, S12-S23.

5. Scheen AJ (2003) Is there a role for alpha-glucosidase inhibitors in the prevention of type 2 diabetes mellitus? Drugs $\mathbf{6 3}$, 933-951.

6. Chiasson JL, Josse RG, Gomis R, et al. (2003) Acarbose treatment and the risk of cardiovascular disease and hypertension in patients with impaired glucose tolerance: the STOP-NIDDM trial. JAMA 290, 486-494.

7. Aston LM (2006) Glycaemic index and metabolic disease risk. Proc Nutr Soc 65, 125-134.

8. Chiasson JL, Josse RG, Gomis R, et al. (2002) Acarbose for prevention of type 2 diabetes mellitus: the STOP-NIDDM randomised trial. Lancet 359, 2072-2077.

9. Collier G \& O'Dea K (1983) The effect of coingestion of fat on the glucose, insulin, and gastric inhibitory polypeptide responses to carbohydrate and protein. Am J Clin Nutr 37, 941-944.

10. Shimotoyodome A, Fukuoka D, Suzuki J, et al. (2009) Coingestion of acylglycerols differentially affects glucose-induced insulin secretion via glucose-dependent insulinotropic polypeptide in C57BL/6J mice. Endocrinology 150, 2118-2126.

11. Díaz EO, Galgani JE \& Aguirre CA (2006) Glycaemic index effects on fuel partitioning in humans. Obes Rev 7, 219-226.

12. Higdon JV \& Frei B (2006) Coffee and health: a review of recent human research. Crit Rev Food Sci Nutr 46, 101-123.

13. Dórea JG \& da Costa TH (2005) Is coffee a functional food? Br J Nutr 93, 773-782.

14. Clifford MN (1999) Chlorogenic acids and other cinnamates -nature, occurrence and dietary burden. J Sci Food Agric 79, $362-372$.

15. Murase T, Misawa K, Minegishi Y, et al. (2011) Coffee polyphenols suppress diet-induced body fat accumulation by downregulating SREBP-1c and related molecules in C57BL/ 6J mice. Am J Physiol Endocrinol Metab 300, E122-E133.

16. Ishikawa A, Yamashita H, Hiemori M, et al. (2007) Characterization of inhibitors of postprandial hyperglycemia from the 
leaves of Nerium indicum. J Nutr Sci Vitaminol (Tokyo) 53 $166-173$.

17. Johnston KL, Clifford MN \& Morgan LM (2003) Coffee acutely modifies gastrointestinal hormone secretion and glucose tolerance in humans: glycemic effects of chlorogenic acid and caffeine. Am J Clin Nutr 78, 728-733.

18. Ida Y, Satoh Y, Ohtsuka M, et al. (1994) Phenolic constituents of Phellodendron amurense bark. Phytochemistry 35, 209-215.

19. Iwai K, Kishimoto N, Kakino $\mathrm{Y}$, et al. (2004) In vitro antioxidative effects and tyrosinase inhibitory activities of seven hydroxycinnamoyl derivatives in green coffee beans. J Agric Food Chem 52, 4893-4898.

20. Kiso T, Hamayasu K, Fujita K, et al. (2003) Inhibition of betafructofuranosidases and alpha-glucosidases by synthetic thio-fructofuranoside. Biosci Biotechnol Biochem 67, $1719-1724$

21. Olthof MR, Hollman PC \& Katan MB (2001) Chlorogenic acid and caffeic acid are absorbed in humans. J Nutr 131, 66-71.

22. Olthof MR, Hollman PC, Buijsman MN, et al. (2003) Chlorogenic acid, quercetin-3-rutinoside and black tea phenols are extensively metabolized in humans. J Nutr 133, 1806-1814.

23. Baur JA, Pearson KJ, Price NL, et al. (2006) Resveratrol improves health and survival of mice on a high-calorie diet. Nature 444, 337-342.

24. Murase T, Nagasawa A, Suzuki J, et al. (2002) Beneficial effects of tea catechins on diet-induced obesity: stimulation of lipid catabolism in the liver. Int J Obes Relat Metab Disord 26, 1459-1464.

25. Greenberg JA, Boozer CN \& Geliebter A (2006) Coffee, diabetes, and weight control. Am J Clin Nutr 84, 682-693.

26. Brownsey RW, Boone AN, Elliott JE, et al. (2006) Regulation of acetyl-CoA carboxylase. Biochem Soc Trans 34, 223-227.

27. Holm C (2003) Molecular mechanisms regulating hormonesensitive lipase and lipolysis. Biochem Soc Trans 31, $1120-1124$
28. Ruderman N \& Prentki M (2004) AMP kinase and malonylCoA: targets for therapy of the metabolic syndrome. Nat Rev Drug Discov 3, 340-351.

29. Bandyopadhyay GK, Yu JG, Ofrecio J, et al. (2006) Increased malonyl-CoA levels in muscle from obese and type 2 diabetic subjects lead to decreased fatty acid oxidation and increased lipogenesis; thiazolidinedione treatment reverses these defects. Diabetes 55, 2277-2285.

30. de Paulis T, Schmidt DE, Bruchey AK, et al. (2002) Dicinnamoylquinides in roasted coffee inhibit the human adenosine transporter. Eur J Pharmacol 442, 215-223.

31. Flatt JP (1995) Use and storage of carbohydrate and fat. $A m J$ Clin Nutr 61, 4 Suppl., 952S-959S.

32. Cagen LM, Deng X, Wilcox HG, et al. (2005) Insulin activates the rat sterol-regulatory-element-binding protein 1c (SREBP-1c) promoter through the combinatorial actions of SREBP, LXR, Sp-1 and NF-Y cis-acting elements. Biochem J 385, 207-216.

33. Hasty AH, Shimano H, Yahagi N, et al. (2000) Sterol regulatory element-binding protein-1 is regulated by glucose at the transcriptional level. J Biol Chem 275, 31069-31077.

34. Eckel RH, Fujimoto WY \& Brunzell JD (1979) Gastric inhibitory polypeptide enhanced lipoprotein lipase activity in cultured preadipocytes. Diabetes 28, 1141-1142.

35. Beck B \& Max JP (1983) Gastric inhibitory polypeptide enhancement of the insulin effect on fatty acid incorporation into adipose tissue in the rat. Regul Pept 7, 3-8.

36. Oben J, Morgan L, Fletcher J, et al. (1991) Effect of the entero-pancreatic hormones, gastric inhibitory polypeptide and glucagon-like polypeptide-1(7-36) amide, on fatty acid synthesis in explants of rat adipose tissue. J Endocrinol 130, 267-272.

37. Miyawaki K, Yamada Y, Ban N, et al. (2002) Inhibition of gastric inhibitory polypeptide signaling prevents obesity. Nat Med 8, 738-742. 